\title{
OncomiR-196 promotes an invasive phenotype in oral cancer through the NME4-JNK-TIMP1-MMP signaling pathway
}

\author{
Ya-Ching Lu ${ }^{1 \dagger}$, Joseph T Chang ${ }^{2 \dagger}$, Chun-Ta Liao ${ }^{3}$, Chung-Jan Kang ${ }^{3}$, Shiang-Fu Huang ${ }^{3}$, I-How Chen ${ }^{3}$, \\ Chi-Che Huang ${ }^{3}$, Yu-Chen Huang ${ }^{4}$, Wen-Ho Chen ${ }^{4}$, Chi-Ying Tsai ${ }^{4}$, Hung-Ming Wang ${ }^{5}$, Tzu-Chen Yen ${ }^{6}$, \\ Guo-Rung You', Chang-Hsu Chiang ${ }^{1}$ and Ann-Joy Cheng ${ }^{1 *}$
}

\begin{abstract}
Background: MicroRNA-196 (miR-196), which is highly up-regulated in oral cancer cells, has been reported to be aberrantly expressed in several cancers; however, the significance of miR-196 in oral cancer has not yet been addressed.

Methods: Cellular functions in response to miR-196 modulation were examined, including cell growth, migration, invasion and radio/chemosensitivity. Algorithm-based studies were used to identify the regulatory target of miR-196. The miR-196 target gene and downstream molecular mechanisms were confirmed by RT-qPCR, western blot, luciferase reporter and confocal microscopy analyses. miR-196 expression was determined in paired cancer and adjacent normal tissues from oral cancer patients.

Results: Both miR-196a and miR-196b were highly over-expressed in the cancer tissue and correlated with lymph node metastasis ( $P=0.001$ and $P=0.006$, respectively). Functionally, miR-196 actively promoted cell migration and invasion without affecting cell growth. Mechanistically, miR-196 performed it's their function by inhibiting NME4 expression and further activating p-JNK, suppressing TIMP1, and augmenting MMP1/9.

Conclusion: miR-196 contributes to oral cancer by promoting cell migration and invasion. Clinically, miR-196a/b was significantly over-expressed in the cancer tissues and correlated with lymph node metastasis. Thus, our findings provide new knowledge of the underlying mechanism of cancer metastasis. miR-196 may serve as a promising marker for better oral cancer management.
\end{abstract}

Keywords: miR-196, Cell invasion, NME4, JNK signaling, Oral cancer, Clinical association

\section{Novelty and impact statement}

In this study, we elucidated the significance of miR-196 in oral cancer. miR-196 promotes cell migration and invasion. Mechanistically, miR-196 exerts these functions by targeting to the NME4 molecule and regulating the downstream JNK-TIMP1-MMP signaling pathway. In addition, both miR-196a and miR-196b were remarkably up-regulated in oral cancer tissues and correlated with

\footnotetext{
* Correspondence: annjoycheng@gap.cgu.edu.tw

${ }^{\dagger}$ Equal contributors

'Department of Medical Biotechnology, College of Medicine, Chang Gung University, 259 Wen-Hwa 1st Road, Taoyuan 333, Taiwan

Full list of author information is available at the end of the article
}

lymph node metastasis. Thus, miR-196 could be a promising marker for better management of oral cancer.

\section{Introduction}

Oral cancer is one of the most prevalent cancers worldwide [1]. Despite improvements in diagnosis and treatment in recent decades, the survival rate for oral cancer has not significantly changed due to the development of distant metastases and therapeutic resistance [2-4]. It is essential to thoroughly investigate the pathogenesis of this disease to provide fundamental knowledge for future clinical applications.

MicroRNAs (miRNAs) constitute an abundant class of small, non-coding RNA molecules that regulate gene

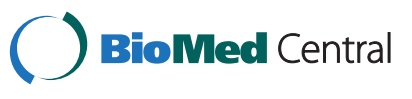


expression by targeting mRNAs to induce translational repression or mRNA degradation [5]. Increasing evidence indicates that miRNAs contribute to the development of cancer by negatively regulating target gene expression, and therefore they can function as tumor suppressors or oncogenes [6,7]. Recently, miRNA screening in several types of cancer has identified unique expression profiles associated with specific tissues or clinical features, including head and neck cancer $[8,9]$. To improve the understanding of the role of miRNAs in oral cancer, we previously performed global miRNA profiling of normal keratinocyte and cancer cell lines. We discovered 23 miRNAs with significantly altered expression in cancer cells, including miR-196 [10]. miR-196 has been reported to be aberrantly expressed in various malignancies, including melanoma, leukemia, and glioblastoma [11-21]. However, the underlying mechanism by which these molecules cause malignancy remains unclear.

In the present study, we characterized the function of miR-196 and elucidated it's molecular mechanism in oral cancer. We found that the miR-196 family positively regulated cell invasion and migration, and had no effect on cell growth. Mechanistically, miR-196 exerted their effects by directly targeting and inhibiting non-metastatic cells 4 (NME4) protein expression to regulate the JNKTIMP1-matrix metalloproteinase (MMP) signaling pathway. We revealed that both miR-196a and miR-196b were highly over-expressed in the cancer tissues of patients with oral cancer, demonstrating the clinical significance of these molecules during cancer progression.

\section{Materials, subjects, and methods}

Cells and cell lines

Four oral cancer cell lines (OECM1, SAS, CGHNC8, and CGHNC9) and two normal keratinocyte cell lines (CGHNK2 and CGHNK4) were used [10]. CGHNK2 and CGHNK4 cells are HPV-immortalized lines of normal keratinocytes that were described previously [10]. The immortalized normal keratinocyte cells were maintained in KSFM medium (Life Technologies, Inc., Gibco BRL, Rockville, MD, USA). The cancer cell lines were grown in 100\% DMEM or RPMI 1640 medium containing $10 \%$ fetal bovine serum (Life Technologies, Inc.). All cells were cultured at $37^{\circ} \mathrm{C}$ in a humidified atmosphere with $5 \% \mathrm{CO}_{2}$.

\section{Cloning and transfection of miR-196-specific plasmids and inhibitory antagomir oligonucleotides}

All the oligonucleotides used in this study, including the specific stem-loop sequences of miR-196a, miR-196b, the inhibitory antagomir oligonucleotides (Anti-196a and Anti-196b), random sequence for antagomir control (RC) are listed in Additional file 1: Table S1. The stem- loop oligonucleotides were inserted into the multiple cloning site of the pcDNA 3.1(+) expression vector (Invitrogen, Carlsbad, CA, USA) to construct the miR196 overexpression plasmids. To promote miR-196 expression, $3 \mu \mathrm{g}$ of miR-196 plasmid was transfected into cells plated in 100-mm dishes. The miR-196a, miR-196b antagomir and the random sequence oligonucleotides for controls were purchased from TRI-I Biotech, Inc. (New Taipei City, Taiwan). To suppress miR-196 expression, $300 \mu \mathrm{M}$ antagomir oligonucleotides were transfected into the cells. Transfection was performed using the Lipofectamine $2000^{\mathrm{Tm}}$ reagent (Invitrogen) in OPTIMEM medium (Invitrogen), and the cells were incubated at $37^{\circ} \mathrm{C}$ in a humidified atmosphere with $5 \% \mathrm{CO}_{2}$ for $10 \mathrm{~h}$, similarly as previously described [22]. Afterward, the medium was replaced with fresh complete medium, and the cells were continuously cultured.

\section{Cell migration assay}

Cell migration was determined using an in vitro wound healing assay as previously described [23]. After transfection of the miR-196 overexpression plasmids or the antagomir oligonucleotides, $3.5 \times 10^{4}$ cells were seeded in ibidi $^{\circledR}$ culture inserts (ibidi LLC, Verona, WI, USA) on top of a 6-well plate. After $8 \mathrm{~h}$ of incubation, the culture inserts were detached to form a cell-free gap in the cell monolayer. After changing to culture medium containing $1 \%$ FCS, cell migration of toward the gap area was photographed every $6 \mathrm{~h}$. All the experiments were performed at least three times independently and that typical results were shown. In each sample, the invasion ability was quantified by comparing the distance of the cell-free gap after normalization to the control group. The error bars shown in the relevant figures indicated the standard deviation of the quantification results in all experiments.

\section{Cell invasion assay}

The invasive abilities of the cells were determined by culturing the cells on a polycarbonate membrane coated with Matrigel (Becton Dickinson Biosciences, Franklin Lakes, NJ, USA) in a Millicell invasion chamber (Millipore, Billerica, MA, USA) as previously described [23]. Briefly, Matrigel was first coated onto the membrane of the Millicell upper chamber for $12 \mathrm{~h}$ at $37^{\circ} \mathrm{C}$. After transfection of the miR-196 overexpression plasmids or antagomir oligonucleotides, the cells were seeded in the upper chamber with 1\% FBS medium. The lower chamber contained complete culture medium (containing $10 \% \mathrm{FBS}$ ) to trap invading cells. After incubation at $37^{\circ} \mathrm{C}$, the cells that invaded through the Matrigel-coated membranes into the lower chamber were stained with crystal violet and photographed. All the experiments were performed at least three times independently and 
that typical results were shown. In each sample, the invasion ability was quantified by comparing the density of crystal violet dye after normalization to the control group. The error bars shown in the relevant figures indicated the standard deviation of the quantification results in all experiments.

\section{mRNA and miRNA analysis by reverse transcription- quantitative PCR (RT-qPCR)}

Total RNA was isolated from cells using TRIzol reagent (Gibco BRL). For mRNA determination, RT was performed as previously described [24]. For miRNA determination, the revere transcription was performed as previously described [10] using miR-196-specific stemloop RT primers and assay kits (ABI, Forest City, CA, USA) according to the manufacturer's suggested protocol. The PCR primers used for target genes are list in Additional file 1: Table S1. The results of real-time PCR, recorded as threshold cycle numbers, were normalized against an internal control (U6 RNA for miRNAs or GAPDH for mRNA). The comparative threshold cycle $(\Delta \Delta \mathrm{Ct})$ method was used to determine the gene expression. All the experiments were performed duplicate for at least three times. The error bars shown in the relevant figures indicated the standard deviation of the quantification results in all experiments.

\section{Protein extraction and western blot analysis}

Protein extraction and western blot analysis were performed as previously described [25]. Briefly, cellular proteins were extracted using lysis buffer by incubating the cells on ice for $30 \mathrm{~min}$. Samples were centrifuged at $14,000 \mathrm{~g}$ for $30 \mathrm{~min}$, and the supernatant was collected. The protein samples were boiled at $95^{\circ} \mathrm{C}$ for $5 \mathrm{~min}$, separated by electrophoresis on $10 \%$ polyacrylamide gels containing $0.1 \%$ SDS, and transferred to nitrocellulose membranes. The membranes were incubated with primary followed by horseradish peroxidase-conjugated secondary antibodies. The primary antibodies used in this study are listed in Additional file 1: Table S2. The membranes were developed using an ECL developing solution (Millipore) followed by autoradiography. All the experiments were performed at least three times independently and that typical results were shown. In each sample, the protein expression shown in each band was quantified after normalization to the GAPDH expression level. The error bars shown in the relevant figures indicated the standard deviation of the quantification results in all experiments.

\section{Luciferase reporter assay for the NME4 3'-UTR}

The pMIR-REPORT firefly luciferase vector plasmid (pMIR, Ambion, Grand Island, NY, USA) was used. The 3'-UTR region of wide-type NME4 was amplified by
PCR and cloned downstream of the luciferase vector (p-UTR-WT). A mutant sequence was also cloned as a validation plasmid (p-UTR-mut). pMIR, p-UTR-WT, and p-UTR-mut was co-transfected with the miR-196specific antagomirs or overexpression plasmids into OECM1 or SAS cells. The pRL-SV vector (Promega, Madison, Wisconsin, USA) containing Renilla luciferase was also transfected for each condition as a reference control. Firefly and Renilla luciferase activities were measured using the Dual-Luciferase Reporter Assay System (Promega) according to the manufacturer's instructions [26]. All the experiments were performed triplicate for at least three times, and the similar results were obtained. The error bars shown in the relevant figures indicated the standard deviation of a triplicate experiment.

\section{Immunofluorescence staining and confocal microscopy}

Immunofluorescence staining and confocal microscopy were performed as previously described [27]. Briefly, cells were seeded onto coverslips coated with poly-Llysine and incubated overnight at $37^{\circ} \mathrm{C}$. After washing, the cells were fixed with formaldehyde, permeabilized with a permeation buffer, and blocked with 1\% FBS. After overnight incubation with primary antibodies, the coverslips were incubated with fluorescence-conjugated secondary antibodies (Molecular Probes, Invitrogen, Carlsbad, CA, USA). The coverslips were then mounted with mounting medium containing DAPI dye (Vector Laboratories, Burlingame, CA, USA), and the fluorescence was visualized using a confocal laser microscope (Leica TCS Sp2 MP).

\section{Patients and clinical association study and statistical analysis}

This study was approved by the Institutional Review Broad of the Human Investigation Committee in Chang Gung Memorial Hospital. Written informed consent was obtained from all patients participating in this study. Fifty-four patients who visited Chang Gung Memorial Hospital (Taoyuan, Taiwan) were recruited for this study. The characteristics of these patients are summarized in Table 1. This study consisted of 5 (9\%) females and 49 (91\%) males. The mean age of the patients was 54.6 years old, with a median age of 53.0 years (range, 35-77 years). A total of 25 patients (46\%) consumed alcohol, 30 patients (56\%) smoked cigarettes, and 37 (68\%) chewed betel quid. Cancer lesions were in oral tongue $(\mathrm{n}=19,35 \%)$, buccal mucosa $(\mathrm{n}=17,32 \%)$, other oral cavity sites $(\mathrm{n}=17,31 \%)$, or soft plate $(\mathrm{n}=1,1.9 \%)$. The surgically dissected cancer tissues and small pieces of adjacent normal counterpart were obtained before chemotherapy or radiotherapy. Twenty patients (37\%) were receiving radical surgery, and $13(24 \%)$ and 21 
Table 1 Characteristics 54 patients with oral cancer, and the association of miR-196a and miR-196b expressions with clinicopathological status

\begin{tabular}{|c|c|c|c|c|}
\hline \multirow[b]{2}{*}{ Characteristics $(n)$} & \multicolumn{2}{|c|}{ miR-196a } & \multicolumn{2}{|c|}{ miR-196b } \\
\hline & low $(n, \%)$ & high $(n, \%)$ & low $(n, \%)$ & high $(n, \%)$ \\
\hline \multicolumn{5}{|l|}{ Sex } \\
\hline Female (5) & $0(0)$ & $5(100)$ & $1(20)$ & $4(80)$ \\
\hline Male (49) & $12(24)$ & $37(76)$ & $18(37)$ & $31(63)$ \\
\hline *P value & 0.210 & & 0.455 & \\
\hline \multicolumn{5}{|l|}{ Age } \\
\hline$\leq 55$ years old $(30)$ & $6(20)$ & $24(80)$ & $9(30)$ & $21(70)$ \\
\hline$>55$ years old $(24)$ & $6(25)$ & $18(75)$ & $10(42)$ & $14(58)$ \\
\hline$P$ value & 0.661 & & 0.372 & \\
\hline \multicolumn{5}{|l|}{ Alcohol drinking } \\
\hline No (29) & $6(21)$ & $23(79)$ & $10(34)$ & $19(66)$ \\
\hline Yes (25) & $6(24)$ & $19(76)$ & $9(36)$ & $16(64)$ \\
\hline$P$ value & 0.770 & & 0.907 & \\
\hline \multicolumn{5}{|l|}{ Cigarette smoking } \\
\hline No (24) & $3(12)$ & $21(88)$ & $7(29)$ & $17(71)$ \\
\hline Yes (30) & $9(30)$ & $21(70)$ & $12(40)$ & $18(60)$ \\
\hline$P$ value & 0.124 & & 0.407 & \\
\hline \multicolumn{5}{|l|}{ Betel quid chewing } \\
\hline No (17) & $3(18)$ & $14(82)$ & $7(41)$ & $10(59)$ \\
\hline Yes (37) & $9(24)$ & $28(76)$ & $12(32)$ & $25(68)$ \\
\hline$P$ value & 0.584 & & 0.532 & \\
\hline \multicolumn{5}{|l|}{ Pathologic T-status } \\
\hline T1-T2 (25) & $6(24)$ & $19(76)$ & $9(36)$ & $16(64)$ \\
\hline T3-T4 (29) & $6(21)$ & $23(79)$ & $10(34)$ & $19(66)$ \\
\hline$P$ value & 0.770 & & 0.907 & \\
\hline \multicolumn{5}{|l|}{ Pathologic N-status } \\
\hline pNo (32) & $12(37)$ & $20(63)$ & $16(50)$ & $16(50)$ \\
\hline $\mathrm{pN}+(22)$ & $0(0)$ & $22(100)$ & $3(14)$ & $19(86)$ \\
\hline *P value & 0.001 & & 0.006 & \\
\hline \multicolumn{5}{|l|}{ Pathologic stage } \\
\hline | - || (19) & $6(32)$ & $13(68)$ & $8(42)$ & $11(58)$ \\
\hline III - IV (35) & $6(17)$ & $29(83)$ & $11(31)$ & $24(69)$ \\
\hline$P$ value & 0.223 & & 0.433 & \\
\hline \multicolumn{5}{|l|}{ Differentiation } \\
\hline Well (15) & $5(33)$ & $10(67)$ & $6(40)$ & $9(60)$ \\
\hline Moderate (35) & $7(20)$ & $28(80)$ & $13(37)$ & $22(63)$ \\
\hline Poor (4) & $0(0)$ & $4(100)$ & $0(0)$ & $4(100)$ \\
\hline${ }^{\#} P$ value & 0.137 & & 0.280 & \\
\hline Total & $12(22)$ & $42(78)$ & $19(35)$ & $35(65)$ \\
\hline
\end{tabular}

*Fisher's exact test was used.

\#Liner-by-linear association test was used.
(39\%) patients were receiving post-operative radiotherapy and concomitant chemoradiotherapy, respectively.

For each tissue, total RNA was extracted and subjected to miR-196a and miR-196b analyses as described previously. To define the relative levels of miR-196 in the clinical samples, the expression level of each tumor sample was normalized to an internal control (U6 RNA) and compared with that of normal tissue from the same patient. The cutoff points were determined after calculating the receiver operating characteristic (ROC) curve for best fit of sensitivity and specificity. Expression levels greater than 15-fold for miR-196a and 7-fold for miR$196 \mathrm{~b}$ in tumor tissues compared to the expression in normal tissue were defined as 'high.' Level. The Pearson chi-square test was used to examine the association of miR-196 expression with clinicopathologic features, including TNM stage. Survival curves were calculated by the Kaplan-Meier method with a log-rank test. All $P$ values were two-sided, and the significance level was set at $P<0.05$.

\section{Results}

Both miR-196a and miR-196b promote cell migration and invasion without affecting cell growth

To determine the carcinogenic functions of miR-196a and miR-196b, in vitro loss-of-function experiments using antagomir oligonucleotides and gain-of-function experiments using miRNA plasmid transfections were performed. The results indicated that the antagomirs against miR-196a and miR-196b substantially inhibited their expression by $79-80$ and $62-73 \%$, respectively, in OECM1 and SAS cells after 1 day (Figure 1A). Plasmid transfection upregulated miR-196a and miR-196b levels by $2.8-5.7$ - and 4.6-7.1-fold, respectively, in OECM1 and SAS cells after 1 day (Figure 1A). The potential effect of miR-196a or miR-196b on cell growth was examined in OECM1 and SAS cells. As shown in Additional file 2: Figure S1, silencing of miR-196a or miR-196b had no effect on cell proliferation. Similarly, over-expression of miR-196a or miR-196b has no significant effect on cell colony growth. These results suggested that miR196 has minimal effect on growth regulation.

The potential effect of miR-196 on and chemo/radiosensitivity was also examined using a clonogenic survival assay. Silencing of miR-196a or miR-196b had no effect on cell survival in response to cisplatin treatment (Additional file 2: Figure S2A). However, miR-196a and miR-196b had differential effects on radiosensitivity. Whereas miR-196b depletion had no effect, both cell lines were significantly more sensitive to radiation after miR-196a silencing (Additional file 2: Figure S2B). This result suggests that miR-196a, but not miR-196b, protects cells against radiation damage. 


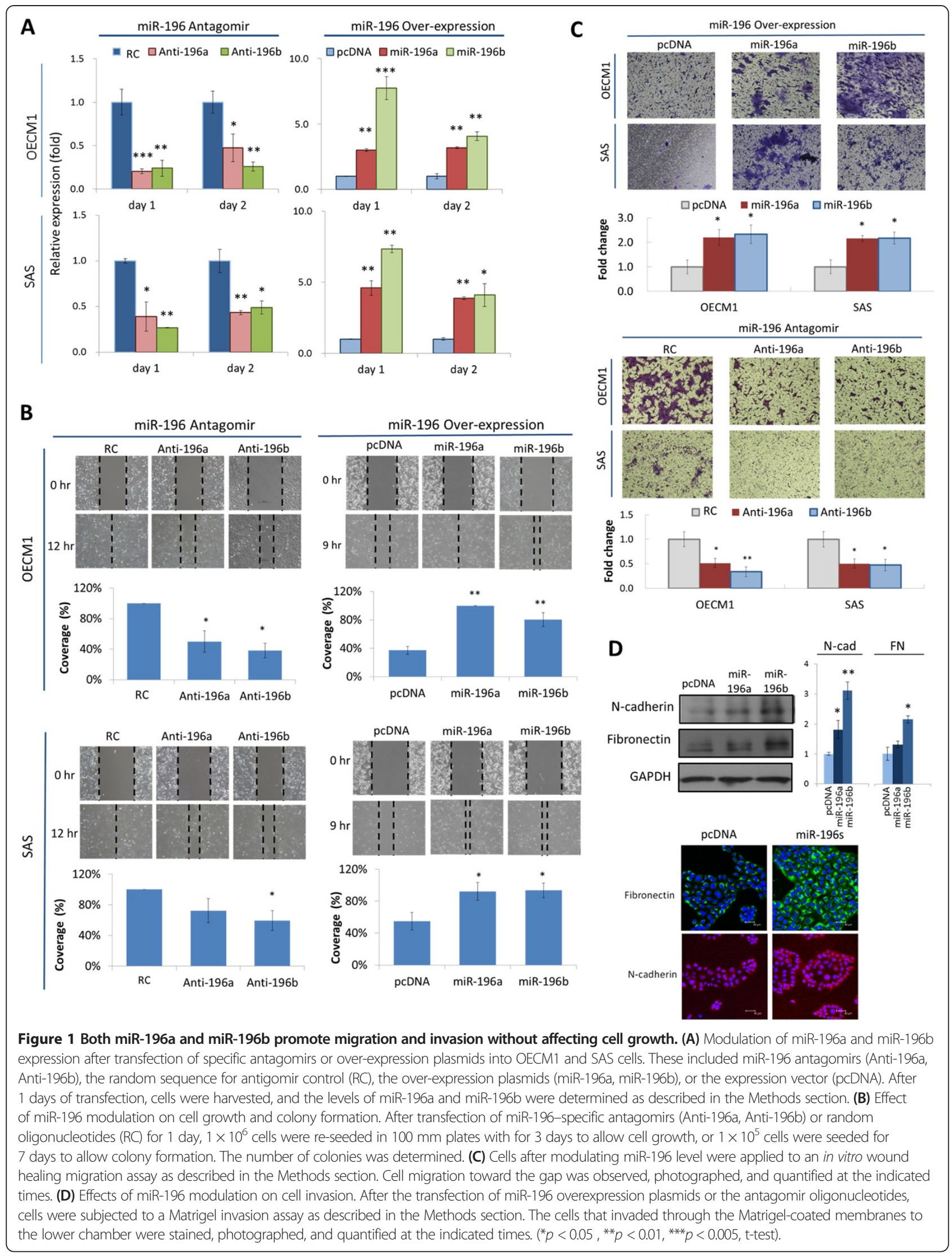


Cell migration and invasion were next analyzed using in vitro wound healing and Matrigel invasion assays. As shown in Figure 1B, miR-196 silencing resulted in slower migration toward the gap area in both OECM1 and SAS cells, with reductions of $28-50 \%$ and $41-62 \%$, respectively, for miR-196a and miR-196b at 12 hours. Consistently, miR-196 over-expression significantly enhanced cell migration in both cell lines, with 1.9-2.7- and 1.72.2-fold increases, respectively, for miR-196a and miR196b at 9 hours. Similar effects were also observed in cell invasion ability (Figure 1C). Depletion of miR-196a or miR-196b dramatically reduced the invading cells by 40-50\% in both OECM1 and SAS cells. Consistently, miR-196a or miR-196b over-expression significantly enhanced cell invasion by 2.2 -fold in both cell lines. Supporting these cellular findings, the expressions of cell adhesion molecules $\mathrm{N}$-cadherin and fibronectin were up-regulated in the miR-196 over-expressing cells (Figure 1D). Collectively, miR-196a and miR-196b promote migration and invasion in oral cancer cells but exhibit minimal effects on cell growth.

\section{NME4 is a direct regulatory target of miR-196}

To identify the potential target of miR-196, computational prediction software, including PicTar, miRanda, and TargetScan, was used. These programs identified common candidates for both miR-196a and miR-196b (Additional file 2: Figure S3). The expression of these genes was further confirmed by RT-qPCR in response to miR-196 modulation. Four genes (ABCB9, HOXA5, MGATA4, and NME4) were upregulated by more than 20\% after miR-196 depletion, whereas three genes (HOXB6, SMCR8, and NME4) were downregulated by more than 20\% after miR-196 overexpression (Additional file 2: Figure S3). Of these genes, only the expression of NME4 changed consistently in both confirmation studies. Therefore, NEM4 is a potential miR-196 regulatory target.

To determine the association of miR-196 and NME4, the expression levels of these molecules were examined in two lines of normal keratinocytes and four oral cancer cell lines. As shown in Figure 2A, miR-196 was significantly up-regulated in all cancer cell lines compared to those in normal cells, with 92- and 71-fold higher in average for miR-196a and miR-196b respectively. By contrast, NME4 expression was reduced in all cancer cell lines at both mRNA (3.9 fold) and protein levels (2.3 fold) (Figure 2B). This reverse correlation between these molecules further suggests that NME4 is a regulatory target of miR-196 (Figure 2C).

To further examine whether NME4 is a down-stream target of miR-196, the potential effect of NME4 protein expression was determined in response to miR-196 modulation. As shown in the Figure 2D, NME4 levels were elevated (1.4-2.9-fold) or reduced (52-78\%) upon
miR-196 silencing or over-expression. To validate the regulatory target of NME4, a luciferase reporter assay was performed. Reporter plasmids that carry human NME4-3'UTR wild-type sequence (p-UTR-WT) and mutant sequence (p-UTR-mut) (Figure 2E) were co-transfected with either miR-196 antagomirs or expression plasmids. Silencing miR-196a or miR-196b increased NME4 wild-type UTR reporter activity in both OECM1 (1.7-2.0-folds) and SAS (1.3-fold) cells but had no effect on mutant UTR or empty vector reporter activity. Consistently, over-expression of miR-196a or miR-196b reduced NME4 wild-type UTR reporter activity both cell lines (by 57\% in OECM1 cells and 29-36\% in SAS cells). However, these miR-196 modulations exhibited minimal effects on mutant UTR reporter activity (Figure 2E). Taken together, these results suggest that NME4 is a down-stream regulatory target of miR-196.

\section{NME4 suppressed the effects of miR-196 on cell migration and invasion}

To investigate whether the enhancement of cell migration and invasion by miR-196 occurred via the suppression of NME4, these cellular effects were analyzed upon exogenous expression of NME4 in miR-196-overexpressing cells. After verifying the expression status of miR-196 and NME4 upon specific plasmid transfection (Figure 3A), cell invasion and migration were examined (Figure 3B-C). MiR-196 transfection significantly promoted cell invasion (2.3-fold) and migration (2.1-fold). However, cell invasion (Figure $3 \mathrm{~B}$ ) and migration (Figure 3C) were inhibited by 39 and 43\%, respectively, upon exogenous NME4 expression. Transfection of NME4 alone had no effect on cell invasion or migration. Hence, the effect of miR-196 on cell migration and invasion is NME4-dependent.

\section{Cellular function of miR-196 occurs through the NME4-JNK- TIMP1-MMP1/9 molecular pathway}

The mitogen-activated protein kinase (MAPK) pathway has been well characterized and demonstrated to play an important role in cell mobility $[28,29]$. We investigated whether the effect of the miR-196-NME4 axis on cellular functions was regulated by MAPK molecules. Possible alterations in the phosphorylation status on three MAPK molecules, JNK, Erk, and p38, were examined by immunoblotting upon the modulation of miR-196 or NME4 expression via plasmid transfection. As shown in Figure 4A, miR-196 and NME4 had minimal effects on phospho-Erk (p-Erk) and phospho-p38 (p-p38) levels. However, phospho-JNK (p-JNK) levels were significantly increased by 2.6 - and 1.8-fold by miR-196a and miR196b modulation, respectively, whereas p-JNK levels were reduced to 0.7 -fold of control levels by NME4 


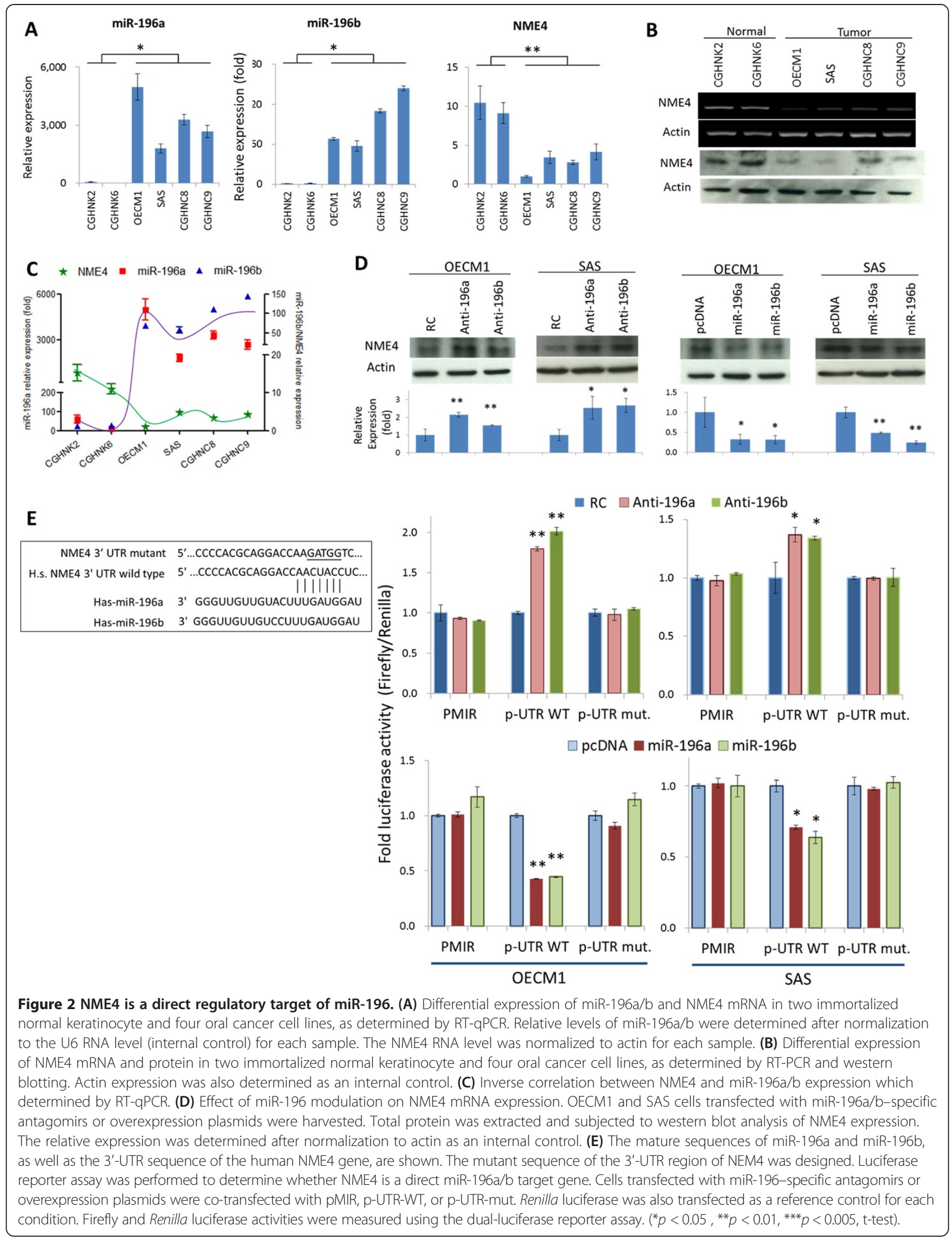



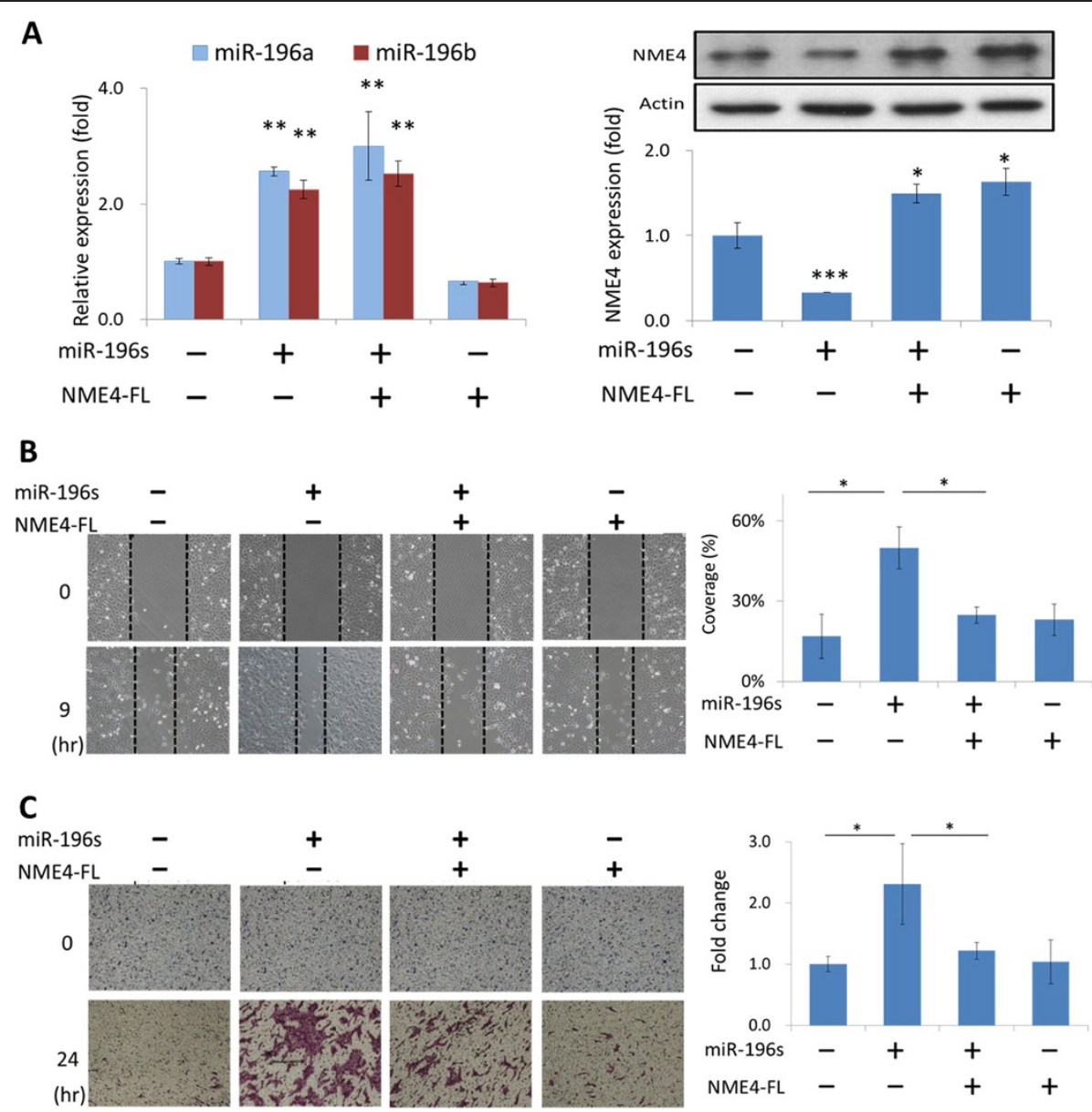

Figure 3 NME4 suppressed the effect of miR-196s on cell migration and invasion. (A) Verification of the expression status of miR-196 s and NME4 after transfection of the miR-196 overexpression or NME4 plasmids. After the transfection of miR-196 and/or NME4 plasmids into OECM1 cells, cell invasion (B) and migration (C) were examined as described in the Methods section. The empty vectors (pcDNA for miR-196 and pCIneo for NME4) were added if necessary to make total DNA amount being equally used in all experiments. $\left({ }^{*} p<0.05,{ }^{* *} p<0.01,{ }^{* * *} p<0.005\right.$, t-test).

modulation. These results suggest that the miR-196NME4 axis stimulates JNK phosphorylation.

The MMP family of proteins (MMP1, MMP2, and MMP9) and their tissue inhibitor TIMP1, which can promote or inhibit the digestion of the extracellular matrix $[30,31]$ were also examined (Figure 4A). Consistently, exogenous expression of miR-196a or miR-196b suppressed TIMP1 expression and enhanced MMP1 and MMP9 expression. Consistently, exogenous NME4 elevated TIMP1 expression but suppressed MMP1 and MMP9 expression. These results suggest that miR-196 promotes cell invasion through the NME4-JNK pathway, leading to the suppression of TIMP1 activity and elevation of MMP1/9 activity.

To determine whether JNK phosphorylation affects MMP expression, the JNK inhibitor SP600125 was used. As shown in Additional file 2: Figure S5, the treatment with SP600125 $(0-50 \mu \mathrm{M})$ suppressed phospho-c-Jun (p-c-Jun) expression with a concomitant increase in
TIMP1 expression and decrease in MMP1/9 expression. These concentration-dependent alterations suggest that TIMP1 and MMP1/9 are downstream targets of p-JNK and p-c-Jun.

To validate the regulation of the miR-196-NME4-pJNKTIMP-MMP pathway, immunofluorescence staining and confocal microscopy were performed. As shown in Figure 4B, exogenous miR-196 reduced NME4 expression and elevated p-JNK and MMP9 expression compared to the findings in the control. On the contrary, exogenous NME4 reduced p-JNK and MMP9 expression. Introduction of NME4 in miR-196-overexpressing cells reversed the effect of miR-196 on p-JNK and MMP9 expression. Furthermore, this molecular pathway was also confirmed in another oral cell line (SAS) (Additional file 2: Figure S6). Taken together, these results suggest that miR-196 exerts it's effect through the NME4-pINK-TIMP1-MMP1/9 pathway (Figure $4 \mathrm{C}$ ). 


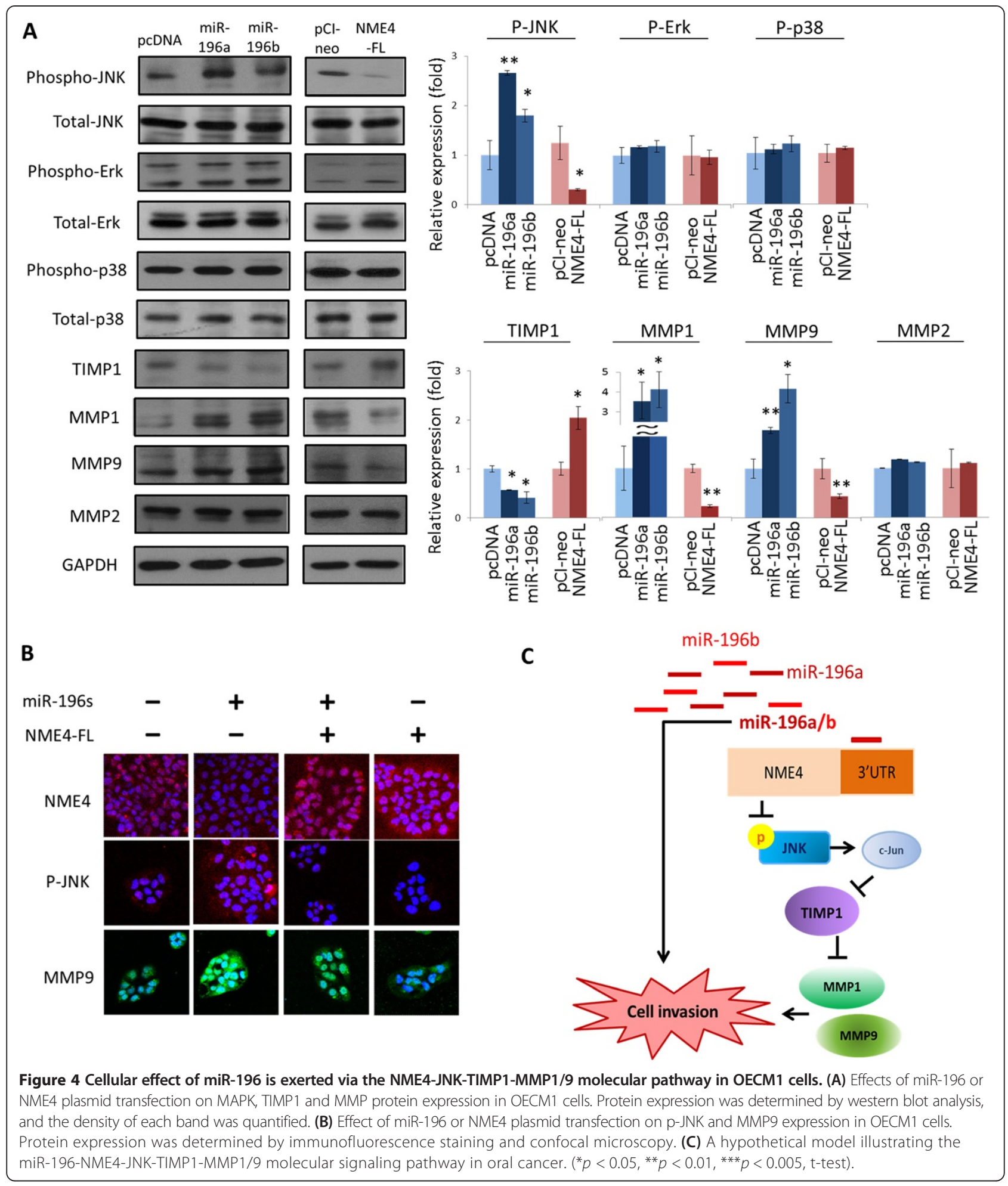

High expression of miR-196a and miR-196b in cancer tissues correlates with the clinical $\mathrm{N}$-stage

To understand the clinical significance of miR-196, normal and cancerous tissues from 54 patients with oral cancer were obtained for analysis. For each tissue sample, the relative miRNA levels were determined, and the results are shown in Figure 5. Both miR-196a and miR-196b were substantially overexpressed in the cancer tissues. Compared to their normal counterparts, 96.3 (52 of 54) and $88.6 \%$ (48 of 54 ) of the cancer tissues exhibited greater 


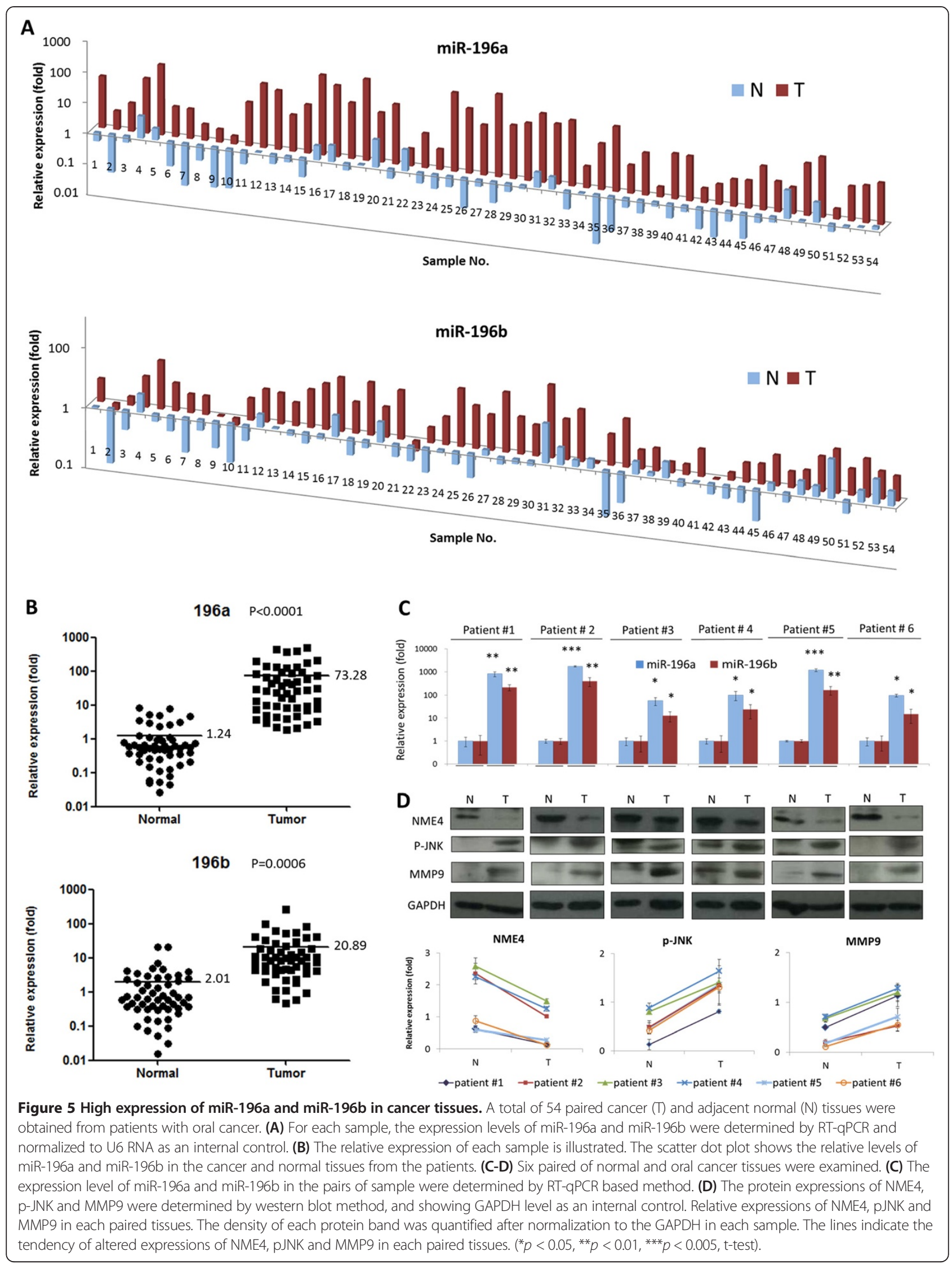


than 2-fold higher expression of miR-196a and miR-196b (Figure 5A), respectively. On average, miR-196a and miR196b levels were elevated by 59.1- and 10.4-fold, respectively, in the cancer tissues (Figure 5B).

To determine miR-196 downstream regulatory mechanism in vivo, six paired normal and cancerous oral cancer tissues were examined. As expected, miR-196a and miR-196b were significantly over-expressed in all cancer tissues (Figure 5C). Consistent with these cellular findings, the NME4 target molecule was substantially suppressed, and an elevation of phosphorylated JNK and MMP9 protein expression was observed (Figure 5D). These results confirmed that the dysregulation pathway of miR-196-NME4-pJNK-MMP molecular axis occurring in oral cancer patients.

To determine the potential association between cancer status and miR-196 expression, Pearson's chi-squared test was used for statistical analysis. The associations of miR-196 expression with cancer stage and pathological status are shown in Table 1. There was no significant correlation of miR-196 expression with the pathological T stage, overall stage, differentiation status, alcohol consumption, cigarette smoking, or betel quid chewing. However, a significant correlation was found between high miR-196 levels and the pathological $\mathrm{N}$ stage $(P=$ 0.001 and $P=0.006$ for miR-196a and miR-196b, respectively). These results indicate the clinical significance of the miR-196 molecules in oral cancer.

\section{Discussion}

The dysregulation of miRNAs is associated with malignant transformation. Previously, miR-196 expression was shown to be altered in several cancers. Although some investigators have reported decreased miR-196 expression in cancers, others have observed increased miR-196 expression. For example, miR-196a and miR-196b are down-regulated (or function as tumor suppressors) in melanoma [11] and acute lymphoblastic leukemia [17,19]. However, miR-196a and miR-196b over-expression has been observed in several malignant diseases, including cancers of the esophagus [21], pancreas [20], colorectum [12], glioblastoma [14], and several types of leukemia $[13,18,32]$. High miR-196a levels have also been associated with a poor prognosis in pancreatic cancer, glioblastoma, and oral squamous cell carcinoma. Furthermore, the polymorphism of pre-miR-196a2 gene was observed in several malignant diseases, including head and neck cancer, and has been associated with cancer susceptibility or prognosis [33-37]. These studies indicate that miR-196 dysregulation plays an important role in carcinogenesis. Consistent with other reports, we previously observed miR-196 overexpression in oral cancer cell lines [10]. In that study, we further identified miR-196 overexpression in the cancer tissues of approximately $90 \%$ of patients with oral cancer compared to their expression in normal tissue (Figure 5), and this overexpression was associated with an aggressive phenotype with lymph node invasion (Table 1). These results demonstrate the significance of miR-196 in the development of oral cancer.

There are limited reports on the role of miR-196 in cancer development. In a study of breast cancer, ectopic miR-196a expression suppressed cell invasion, but the expression level of miR-196 was not correlated with the clinical metastatic status [15]. This result is different from the findings in gastrointestinal cancers, in which miR-196 overexpression promotes cell migration and invasion in colorectal and gastric cancer cells [12,38,39]. In another study, transfection of miR-196a mimic oligonucleotides into esophagus cells revealed that miR-196a promoted cell proliferation and suppressed apoptosis [40]. Thus far, the function of miR-196 in cancer remains obscure. In this study, we determined the role of miR-196 in oral cancer using both silencing and overexpression approaches. We found that both miR-196a and miR-196b actively promoted cell migration and invasion (Figure 1B-C), which were supported by the altered expression of fibronectin and N-cadherin (Figure 1D). However, neither miR-196a nor miR-196b affected cell growth and colony formation (Additional file 2: Figure S1). Hence, the main function of the miR-196 family in oral cancer is the regulation of cell mobility and invasiveness.

Identification of the target molecules of these miRNAs is of high interest. Previously, several molecules were reported as regulatory targets of miR-196. HOX family members have been reported primarily, including HOXB7 (miR-196a target) in melanoma [16], HOX-A (miR196b target) in acute lymphoblastic leukemia [18], and HOX-C8 (miR-196 family target) in melanoma [11] and breast cancer [15]. In this study, four HOX family genes (HOXA5, HOXB6, HOXB7, HOXC8) were identified as targets of miR-196. However, none of these genes responded to miR-196 perturbation (Additional file 2: Figure S4). This difference may be due to the distinct tissue specificity among the various regulatory targets of miR-196. In this study, after clarifying the correlation between NME4 and miR-196 expression in cells (Figure 2A-C), assessing the response of NME4 to miR-196 modulation (Figure 2D), we identified NME4 as a direct target of miR-196a and miR-196b in oral cancer using a luciferase reporter assay (Figure 2E),

NME4, also named nm23-H4, is a member of the nm23 family [41]. The proteins in this family possess nucleoside diphosphate kinase activity, which is believed to be involved in DNA repair mechanisms [42]. Nm23 family proteins also contain the RGD domain, as they can bind to integrin, and this family has been postulated to be involved in cell adhesion and migration [43]. Thus 
far, only a few studies assessed the association of NME4 with cancer, but genomic aberration or altered gene expression has been observed for NME4 in several types of cancers [44-47]. Although the function of NME4 is unclear, it was reported that an nm23 family member, NEM1, is regulated by TP53 [48] and that it acts as a metastatic suppressor [49]. In this study, we also found that ectopic expression of NME4 has no significant effect on cell invasion and migration (Figure 3B-C), indicating that a certain level of NME4 protein is sufficient for maintaining cellular mobility. However, restoration of silenced NME4 suppressed these effects induced by miR-196 (Figure 3B-C), suggesting that NME4 participates in the miR-196 regulatory pathway by inhibiting these functions. Collectively, miR-196 plays an oncogenic role by degrading NME4, thus accelerating cell migration and invasion.

The downstream regulatory mechanism of the miR-196NME4 interaction was further investigated. In examining three MAPK family molecules, we found that $\mathrm{p}-\mathrm{JNK}$, but not p-Erk or p-p38, responded to miR-196 expression and NME4 inhibition, whereas miR-196 and NME4 had minimal effects on the expression of MAPK proteins (Figure 4A). These results indicate that miR-196-NME4 signaling could result in JNK phosphorylation and activation. In addition, TIMP1 and MMP1/9 displayed opposite responses to miR-196 suppression and NME4 augmentation (Figure 4A). These results suggest that TIMP1 and MMP1/9 are the downstream regulatory molecules of the miR-196-NME4 signaling axis. Additionally, we found that p-JNK inhibition increased TIMP1 expression and decreased MMP1/9 expression (Additional file 2: Figure S5). Hence, TIMP1 and MMP1/9 could be regulated by JNK phosphorylation. Moreover, the role of the NME4-pJNKTIMP1-MMP1/9 signaling pathway in miR-196 function was further demonstrated by immunofluorescence staining and confocal microscopy (Figure 4B). Furthermore, this molecular pathway was also confirmed in another oral cell line (SAS) (Additional file 2: Figure S6) and paired normal and cancerous oral cancer tissues (Figure 5D). Thus, miR196 appear to fine-tune the invasion mechanism in oral cancer by inhibiting NME4, leading to the activation of $\mathrm{p}$ JNK and MMP1/9 and suppression of TIMP1 (Figure 4C).

In conclusion, we clarified that miR-196 promotes invasive and migratory phenotypes in oral cancer. Mechanistically, miR-196 exerted its functions by targeting to NME4, leading to the regulation of downstream molecules, including activating $\mathrm{p}-\mathrm{JNK}$, suppressing TIMP1, and augmenting MMP1/9. Consistently, clinical studies have revealed that both miR-196a and miR-196b are remarkably up-regulated in cancer tissue and correlated with lymph node metastasis. Thus, our findings provide new knowledge of the underlying mechanism of cancer metastasis. miR-196 may serve as a promising marker for better oral cancer management.

\section{Additional files}

Additional file 1: Table S1. Names and sequences of oligonucleotides of miR-196 stem-loop, the specific antigomirs and RT-PCR primers. Table S2. The primary antibody used in this study.

Additional file 2: Figure S1. Both miR-196a and miR-196b has minimal effect on cell growth and colony formation in OECM1 and SAS cells. (A) After inhibition of miR-196s by antagomirs, cells were re-seeded to allow cell growth, and cell numbers were measured. (B) Cells were transfected with miR-196s over-expression plasmid to increase miR-196s expression. Clonogenic assay were performed and the number of colonies was determined. Figure S2. Neither miR-196a nor miR-196b affected chemosensitivity, whereas only miR-196a protects cells against irradiation (A) Chemoresistance was determined by the relative number of surviving cells after treatment with serial concentrations $(0-5 \mu \mathrm{g} / \mathrm{ml})$ of cisplatin for 2 days. In each sample, cell survival was counted and compared to the number of surviving untreated cell. (B) Radioresistance was determined using a clonogenic survival assay after treatment with various doses (0-6 Gy) of radiation for 7 days. Figure S3. Bioinformatic prediction of the regulatory targets of miR-196s using PicTar, TargetScan, and miRanda computational prediction software. The shared genes predicted by the three different prediction methods are listed in the table. Figure S4. Validation of the predicted genes by RT-qPCR. After transfection of the miR-196 antagomirs or overexpression plasmids into OECM1 cells, the mRNA level of each gene was determined by RT-qPCR. Relative expression was determined after each gene was normalized to the internal control (Actin). Figure S5. Effect of the JNK phosphorylation inhibitor SP600125 on TIMP1 and MMP protein expression in OECM1 cells. Protein expression was determined by western blot. Figure S6. (A) Effects of miR-196s or NME4 plasmid transfection on p-JNK, MMP1 and MMP9 in SAS cells. (B) Effect of the JNK phosphorylation inhibitor SP600125 on MMP protein expression in SAS cells. Protein expression was determined by western blot.

\section{Competing interests}

The authors declare that they have no competing interests.

\section{Authors' contributions}

YCL performed all experiments; she involved in the design of study, developing assay methodology, acquisition and analysis of laboratory data, and writing the manuscript. JTC participated in the design of study, acquisition of clinical data, performing clinicopathologic analysis, discussion of the laboratory data and revision of manuscript. CTL, CJK, SFH, IHC, CCH, and $\mathrm{YCH}$ participated in acquisition of clinical data and clinicopathologic analysis. CYT, HMW and TCY involved in data analysis, discussion and interpretation. GRY and CHC participated in the design of the study, developing assay methodology, and performed statistical analysis. AJC is the corresponding author as well as the study supervisor; she involved in conception and design of this work, analysis and interpretation of data, writing and revision of the manuscript. All authors have read and approved the final manuscript.

\section{Acknowledgements}

This work was supported by grants from Chang Gung Memorial Hospital (CMRPG300062, CMRPG3A0891, CMRPD190394), and National Science Counsel (NSC-101-2320-B- 182-041-MY3).

\section{Author details}

${ }^{1}$ Department of Medical Biotechnology, College of Medicine, Chang Gung University, 259 Wen-Hwa 1st Road, Taoyuan 333, Taiwan. ${ }^{2}$ Department of Radiation Oncology, Chang Gung Memorial Hospital, Taoyuan, Taiwan. 3.Department of Otorhinolaryngology, Head Neck Surgery, Chang Gung Memorial Hospital, Taoyuan 333, Taiwan. ${ }^{4}$ Department of Oral and maxillofacial Surgery, Chang Gung Memorial Hospital, Taoyuan 333, Taiwan. ${ }^{5}$ Department of Medical Oncology, Chang Gung Memorial Hospital, Taoyuan 333, Taiwan. ${ }^{6}$ Molecular Imaging Center and Department of Nuclear Medicine, Chang Gung Memorial Hospital, Taoyuan 333, Taiwan.

Received: 29 May 2014 Accepted: 11 September 2014

Published: 19 September 2014 


\section{References}

1. Warnakulasuriya S: Global epidemiology of oral and oropharyngeal cancer. Oral Oncol 2009, 45:309-316.

2. Chen YJ, Chang JT, Liao CT, Wang HM, Yen TC, Chiu CC, Lu YC, Li HF, Cheng AJ: Head and neck cancer in the betel quid chewing area: recent advances in molecular carcinogenesis. Cancer Sci 2008, 99:1507-1514.

3. Stucken E, Weissman J, Spiegel JH: Oral cavity risk factors: experts' opinions and literature support. J Otolaryngol 2010, 39:76-89.

4. Saman DM: A review of the epidemiology of oral and pharyngeal carcinoma: update. Head Neck Oncol 2012, 4:1.

5. Garzon R, Marcucci G, Croce CM: Targeting microRNAs in cancer: rationale, strategies and challenges. Nat Rev Drug Discov 2010, 9:775-789.

6. D'Amato NC, Howe EN, Richer JK: MicroRNA regulation of epithelial plasticity in cancer. Cancer Lett 2013, 341:46-55.

7. Ahmad J, Hasnain SE, Siddiqui MA, Ahamed M, Musarrat J, Al-Khedhairy AA: MicroRNA in carcinogenesis \& cancer diagnostics: a new paradigm. Indian J Med Res 2013, 137:680-694.

8. Hui AB, Lenarduzzi M, Krushel T, Waldron L, Pintilie M, Shi W, Perez-Ordonez B, Jurisica I, O'Sullivan B, Waldron J, Gullane P, Cummings B, Liu FF: Comprehensive MicroRNA profiling for head and neck squamous cell carcinomas. Clin Cancer Res 2010, 16:1129-1139.

9. Ramdas L, Giri U, Ashorn CL, Coombes KR, El-Naggar A, Ang KK, Story MD: miRNA expression profiles in head and neck squamous cell carcinoma and adjacent normal tissue. Head Neck 2009, 31:642-654.

10. Lu YC, Chen YJ, Wang HM, Tsai CY, Chen WH, Huang YC, Fan KH, Tsai CN, Huang SF, Kang CJ, Chang JT, Cheng AJ: Oncogenic Function and Early Detection Potential of miRNA-10b in Oral Cancer as Identified by microRNA Profiling. Cancer Prev Res 2012, 5:665-674.

11. Mueller DW, Bosserhoff AK: MicroRNA miR-196a controls melanomaassociated genes by regulating HOX-C8 expression. Int I Cancer 2011, 129:1064-1074.

12. Schimanski CC, Frerichs K, Rahman F, Berger M, Lang H, Galle PR, Moehler M, Gockel I: High miR-196a levels promote the oncogenic phenotype of colorectal cancer cells. World J Gastroenterol 2009, 15:2089-2096.

13. Popovic R, Riesbeck LE, Velu CS, Chaubey A, Zhang J, Achille NJ, Erfurth FE, Eaton K, Lu J, Grimes HL, Chen J, Rowley JD, Zeleznik-Le NJ: Regulation of mir-196b by MLL and its overexpression by MLL fusions contributes to immortalization. Blood 2009, 113:3314-3322.

14. Guan Y, Mizoguchi M, Yoshimoto K, Hata N, Shono T, Suzuki SO, Araki Y, Kuga D, Nakamizo A, Amano T, Ma X, Hayashi K, Sasaki T: MiRNA-196 is upregulated in glioblastoma but not in anaplastic astrocytoma and has prognostic significance. Clin Cancer Res 2010, 16:4289-4297.

15. Li Y, Zhang M, Chen H, Dong Z, Ganapathy V, Thangaraju M, Huang S: Ratio of miR-196 to HOXC8 messenger RNA correlates with breast cancer cell migration and metastasis. Cancer Res 2010, 70:7894-7904.

16. Braig S, Mueller DW, Rothhammer T, Bosserhoff AK: MicroRNA miR-196a is a central regulator of HOX-B7 and BMP4 expression in malignant melanoma. Cell Mol Life Sci 2010, 67:3535-3548.

17. Bhatia S, Kaul D, Varma N: Potential tumor suppressive function of miR-196b in B-cell lineage acute lymphoblastic leukemia. Mol Cell Biochem 2010, 340:97-106

18. Schotte D, Lange-Turenhout EA, Stumpel DJ, Stam RW, Buijs-Gladdines JG, Meijerink JP, Pieters R, Den Boer ML: Expression of miR-196b is not exclusively MLL-driven but is especially linked to activation of HOXA genes in pediatric acute lymphoblastic leukemia. Haematologica 2010, 95:1675-1682.

19. Bhatia S, Kaul D, Varma N: Functional genomics of tumor suppressor miR-196b in T-cell acute lymphoblastic leukemia. Mol Cell Biochem 2011, 346:103-116.

20. Kong X, Du Y, Wang G, Gao J, Gong Y, Li L, Zhang Z, Zhu J, Jing Q, Qin Y, Li Z: Detection of differentially expressed microRNAs in serum of pancreatic ductal adenocarcinoma patients: miR-196a could be a potential marker for poor prognosis. Dig Dis Sci 2011, 56:602-609.

21. Haiart S, Watson DI, Leong MP, Astill D, Bright T, Hussey DJ: MicroRNA-196a \& microRNA-101 expression in Barrett's oesophagus in patients with medically and surgically treated gastro-oesophageal reflux. BMC Res Notes 2011, 4:41.

22. Lin TY, Chang JT, Wang HM, Chan SH, Chiu CC, Lin CY, Fan KH, Liao CT, Chen IH, Liu TZ, Li HF, Cheng AJ: Proteomics of the radioresistant phenotype in head-and-neck cancer: Gp96 as a novel prediction marker and sensitizing target for radiotherapy. Int J Radiat Oncol Biol Phys 2010, 78:246-256
23. Chiu CC, Lin CY, Lee LY, Chen YJ, Lu YC, Wang HM, Liao CT, Chang JT, Cheng AJ: Molecular chaperones as a common set of proteins that regulate the invasion phenotype of head and neck cancer. Clin Cancer Res 2011, 17:4629-4641.

24. Chen YJ, Liao CT, Chen PJ, Lee LY, Li YC, Chen IH, Wang HM, Chang JT, Chen $L$, Yen TC, Tang CY, Cheng AJ: Downregulation of Ches1 and other novel genes in oral cancer cells chronically exposed to areca nut extract. Head Neck 2011, 33:257-266.

25. Lin CY, Lin TY, Wang HM, Huang SF, Fan KH, Liao CT, Chen IH, Lee LY, Li YL, Chen YJ, Cheng AJ, Chang JT: GP96 is over-expressed in oral cavity cancer and is a poor prognostic indicator for patients receiving radiotherapy. Radiat Oncol 2011, 6:136.

26. Chen YJ, Lee LY, Chao YK, Chang JT, Lu YC, Li HF, Chiu CC, Li YC, Li YL, Chiou JF, Cheng AJ: DSG3 facilitates cancer cell growth and invasion through the DSG3-plakoglobin-TCF/LEF-Myc/cyclin D1/MMP signaling pathway. PLoS One 2013, 8:e64088.

27. Chiu CC, Lee LY, Li YC, Chen YJ, Lu YC, Li YL, Wang HM, Chang JT, Cheng AJ: Grp78 as a therapeutic target for refractory head-neck cancer with CD24(-)CD44(+) stemness phenotype. Cancer Gene Ther 2013, 20:606-615.

28. Reddy KB, Nabha SM, Atanaskova N: Role of MAP kinase in tumor progression and invasion. Cancer Metastasis Rev 2003, 22:395-403.

29. Keshet $Y$, Seger $R$ : The MAP kinase signaling cascades: a system of hundreds of components regulates a diverse array of physiological functions. Methods Mol Biol 2010, 661:3-38.

30. Clark IM, Swingler TE, Sampieri CL, Edwards DR: The regulation of matrix metalloproteinases and their inhibitors. Int I Biochem Cell Biol 2008, 40:1362-1378.

31. Bourboulia D, Stetler-Stevenson WG: Matrix metalloproteinases (MMPs) and tissue inhibitors of metalloproteinases (TIMPs): Positive and negative regulators in tumor cell adhesion. Semin Cancer Biol 2010, 20:161-168.

32. Coskun E, von der Heide EK, Schlee C, Kuhnl A, Gokbuget N, Hoelzer D, Hofmann WK, Thiel E, Baldus CD: The role of microRNA-196a and microRNA-196b as ERG regulators in acute myeloid leukemia and acute T-lymphoblastic leukemia. Leuk Res 2011, 35:208-213.

33. Hoffman AE, Zheng T, Yi C, Leaderer D, Weidhaas J, Slack F, Zhang Y, Paranjape T, Zhu Y: microRNA miR-196a-2 and breast cancer: a genetic and epigenetic association study and functional analysis. Cancer Res 2009, 69:5970-5977.

34. Akkiz H, Bayram S, Bekar A, Akgollu E, Ulger Y: A functional polymorphism in pre-microRNA-196a-2 contributes to the susceptibility of hepatocellular carcinoma in a Turkish population: a case-control study. J Viral Hepat 2011, 18:e399-e407.

35. Zhan JF, Chen LH, Chen ZX, Yuan YW, Xie GZ, Sun AM, Liu Y: A functional variant in microRNA-196a2 is associated with susceptibility of colorectal cancer in a Chinese population. Arch Med Res 2011, 42:144-148.

36. Liu CJ, Tsai MM, Tu HF, Lui MT, Cheng HW, Lin SC: miR-196a overexpression and miR-196a2 gene polymorphism are prognostic predictors of oral carcinomas. Ann Surg Oncol 2013, 20(Suppl 3):S406-S414

37. Christensen BC, Avissar-Whiting M, Ouellet LG, Butler RA, Nelson HH, McClean $M D$, Marsit CJ, Kelsey KT: Mature microRNA sequence polymorphism in MIR196A2 is associated with risk and prognosis of head and neck cancer. Clin Cancer Res 2010, 16:3713-3720.

38. Liao YL, Hu LY, Tsai KW, Wu CW, Chan WC, Li SC, Lai CH, Ho MR, Fang WL, Huang KH, Lin WC: Transcriptional regulation of miR-196b by ETS2 in gastric cancer cells. Carcinogenesis 2012, 33:760-769.

39. Niinuma T, Suzuki H, Nojima M, Nosho K, Yamamoto H, Takamaru $H$, Yamamoto E, Maruyama R, Nobuoka T, Miyazaki Y, Nishida T, Bamba T, Kanda T, Ajioka Y, Taguchi T, Okahara S, Takahashi H, Nishida Y, Hosokawa M, Hasegawa T, Tokino T, Hirata K, Imai K, Toyota M, Shinomura Y: Upregulation of miR-196a and HOTAIR drive malignant character in gastrointestinal stromal tumors. Cancer Res 2012, 72:1126-1136.

40. Luthra R, Singh RR, Luthra MG, Li YX, Hannah C, Romans AM, Barkoh BA Chen SS, Ensor J, Maru DM, Broaddus RR, Rashid A, Albarracin CT: MicroRNA-196a targets annexin A1: a microRNA-mediated mechanism of annexin A1 downregulation in cancers. Oncogene 2008, 27:6667-6678.

41. Milon L, Rousseau-Merck MF, Munier A, Erent M, Lascu I, Capeau J, Lacombe ML: nm23-H4, a new member of the family of human nm23/nucleoside diphosphate kinase genes localised on chromosome 16p13. Hum Genet 1997, 99:550-557.

42. Postel EH: Multiple biochemical activities of NM23/NDP kinase in gene regulation. J Bioenerg Biomembr 2003, 35:31-40. 
43. Negroni A, Venturelli D, Tanno B, Amendola R, Ransac S, Cesi V, Calabretta B, Raschella G: Neuroblastoma specific effects of DR-nm23 and its mutant forms on differentiation and apoptosis. Cell Death Differ 2000, 7:843-850.

44. Patocs A, Zhang L, Xu Y, Weber F, Caldes T, Mutter GL, Platzer P, Eng C: Breast-cancer stromal cells with TP53 mutations and nodal metastases. N Engl J Med 2007, 357:2543-2551.

45. Xu ZY, Chen JS, Shu YQ: Gene expression profile towards the prediction of patient survival of gastric cancer. Biomed Pharmacother 2010, 64:133-139.

46. Skotheim Rl, Autio R, Lind GE, Kraggerud SM, Andrews PW, Monni O, Kallioniemi O, Lothe RA: Novel genomic aberrations in testicular germ cell tumors by array-CGH, and associated gene expression changes. Cell Oncol 2006, 28:315-326.

47. Seifert M, Welter C, Mehraein Y, Seitz G: Expression of the $n m 23$ homologues $\mathrm{nm} 23-\mathrm{H} 4, \mathrm{~nm} 23-\mathrm{H} 6$, and $\mathrm{nm} 23-\mathrm{H} 7$ in human gastric and colon cancer. J Pathol 2005, 205:623-632

48. Chen SL, Wu YS, Shieh HY, Yen CC, Shen JJ, Lin KH: P53 is a regulator of the metastasis suppressor gene Nm23-H1. Mol Carcinog 2003, 36:204-214.

49. Marshall JC, Collins J, Marino N, Steeg P: The Nm23-H1 metastasis suppressor as a translational target. Eur J Cancer 2010, 46:1278-1282.

doi:10.1186/1476-4598-13-218

Cite this article as: Lu et al: OncomiR-196 promotes an invasive phenotype in oral cancer through the NME4-JNK-TIMP1-MMP signaling pathway. Molecular Cancer 2014 13:218.

\section{Submit your next manuscript to BioMed Central and take full advantage of:}

- Convenient online submission

- Thorough peer review

- No space constraints or color figure charges

- Immediate publication on acceptance

- Inclusion in PubMed, CAS, Scopus and Google Scholar

- Research which is freely available for redistribution 